\title{
Factores asociados a lesiones preneoplásicas de cuello uterino en usuarias de la ESE del municipio de Aipe, en el periodo enero a sep- tiembre de 2009
}

\section{Factors associated with cervical preneoplastic injuries in patients from state-owned medical insti- tution in Aipe, from january to september, 2009}

\section{Resumen}

Eduardo Mahecha ${ }^{1}$, Sara A. Yaguar ${ }^{1}$, Glitza I. Calderón ${ }^{1}$

El cáncer cervicouterino causa alrededor de 500.000 muertes al año en el mundo, en los países en desarrollo, su incidencia llega hasta 40 por 100.000 mujeres.

Se realizó un estudio epidemiológico observacional, analítico de tipo casos y controles; basado en los casos. En 198 mujeres mayores de edad hasta de 55 años de edad, que acudieron al programa de detección temprana del cáncer cervicouterino en Aipe, Huila del $1^{\circ}$ de enero al 30 de septiembre de 2009. En el estudio se excluyeron todas las mujeres con histerectomía por cualquier causa y aquéllas que fuesen menores de edad o que tuviesen más de 55 años de edad. La población fue entrevistada, con relación a los antecedentes ginecológicos, obstétricos y socioculturales.

El riesgo de presentar lesiones preneoplásicas en el útero, se incrementa en 5,92 veces más en comparación de las que no han presentado infección. El tener de 6 a 7 compañero sexual se incrementa en 4,67 veces más en comparación a las que han tenido un solo compañero sexual.

Palabras clave: Las mujeres que han presentado infecciones vaginales tienen un riego mayor en 5,88 veces en comparación de las que no han presentado dicha enfermedad, al igual que el tener más de cinco compañeros sexuales incrementa el riesgo de cáncer cervicouterino en 20,67 veces más en comparación con las que han presentado de 2 a 4 compañeros sexuales.

1 Especialistas en Epidemiología, Facultad de Salud, Universidad Surcolombiana.

Correspondencia: Eduardo Mahecha Correo electrónico: edmahecha97@gmail.com

Recibido: 24 de marzo de 2011 Aceptado: 29 de abril de 2011

\begin{abstract}
Cervical cancer causes about 500,000 deaths per year worldwide in developing countries; its incidence reaches up to 40 per 100,000 women. An observational, epidemiological, analytical study of cases and controls was carried out in 198 adult women whose age is no more than 55, who attended the cervical cancer early detection program in Aipe, Huila, from January 1 to September 30, 2009. All women with hysterectomy from any cause and those who were minors or who are over 55 years old were excluded from the study. The population was interviewed in relation to gynecological, obstetric and socio-cultural history

The risk of precancerous lesions in the uterus, is increased 5.92 times more in comparison to those women free from infection, having from 6 to
\end{abstract}


7 sexual partners increases 4.67 times more, compared to those who have had a single sexual partner.

Women who have developed vaginal infections have 5.88 times more risk, compared to those who have not suffered from that disease; likewise, having more than five sexual partners increases the risk of cervical cancer 20.67 times more, compared to those who have had from 2 to 4 sexual partners.

\section{Introducción}

En la Revista Colombiana de Obstetricia y Ginecología, "estima que el cáncer cervicouterino causa alrededor de 500.000 muertes al año en el mundo, en los países en desarrollo, su incidencia llega hasta 40 por 100.000 mujeres"(1). Sin embargo, "el cáncer de cuello uterino continúa siendo un problema importante de salud pública pues representa la segunda causa de muerte por cáncer en mujeres en Colombia (Tasa de mortalidad ajustada por subregistro de 18,1 por 100000 mujeres)"(2). Probablemente factores relacionados con barreras de acceso, con una falta de adecuación de los servicios de salud a la forma como se distribuye el riesgo en la población de mujeres, además con la estructura y organización del actual Sistema General de Seguridad Social en Salud (SGSSS) parecen explicar este hecho.

\section{Factores de riesgo para cáncer de cuello uterino}

"El cáncer de cuello uterino y las lesiones premalignas se comportan como una enfermedad de transmisión sexual, asociada especialmente a la infección por el virus del papiloma humano (VPH), aunque existe cierta susceptibilidad genética entre las mujeres con presencia simultánea de HLA-B*07 y HLA-B*0302, pero con disminución del riesgo entre quienes tienen HLADRB1*1301"(3).

\section{Número de compañeros sexuales}

Existe una relación directamente proporcional entre el riesgo de lesión intraepitelial y el número de parejas sexuales. "Esta exposición se ha relacionado básicamente con la probabilidad de exposición al VPH"(4). Por ejemplo, "las mujeres solteras, viudas o separadas tienen más riesgo de infectarse por VPH dado que tienen más compañeros sexuales, sea permanentes u ocasionales"(5).

\section{Enfermedades de transmisión sexual}

Se ha demostrado la asociación de cáncer de cuello uterino con enfermedades de transmisión sexual tipo sífilis o blenorragia, así como historia de infección a repetición del aparato genital; sin embargo, no se ha demostrado relación con antecedentes de infección por Chlamydia trachomatiso herpes simplex. En un estudio realizado en tres países se encontró asociación entre infección por VPH y C. trachomatisen Colombia y España, pero no en Brasil, mientras que otros estudios han asociado la infección del serotipo $G$ de esta bacteria con el virus.

Adicionalmente, la coinfección con "el virus de la inmunodeficiencia humana (VIH) facilita el desarrollo de la neoplasia, con un aumento del riesgo de cáncer entre las infectadas por el VIH de hasta 3,2 veces el riesgo de las mujeres sin $\mathrm{VIH}^{\prime \prime(6)}$.

\section{Materiales y métodos}

Se realizó un estudio epidemiológico observacional, analítico de tipo casos y controles; "basado en los casos"(7); los casos se identifican como los individuos a quienes se les diagnóstico la enfermedad de interés, en 198 mujeres mayores de edad hasta de 55 años de edad, que acudieron al programa nacional de detección temprana del cáncer cervicouterino en el municipio de Aipe del $1^{\circ}$ de enero al 30 de septiembre de 2009, en el estudio se excluyeron todas las mujeres con histerectomía por cualquier causa y aquéllas que fuesen menores de edad o que tuviesen más de 55 años de edad, la pobla- 
Tabla 1.

\begin{tabular}{|c|c|c|c|c|c|c|c|c|}
\hline \multirow{2}{*}{$\begin{array}{l}\text { Variable } \\
\text { Infección vaginal }\end{array}$} & \multicolumn{2}{|c|}{ Casos } & \multicolumn{2}{|c|}{ Controles } & \multirow[t]{2}{*}{ OR } & \multirow[t]{2}{*}{ IC $95 \%$} & \multirow{2}{*}{$\begin{array}{c}\text { Chi }^{2} \\
\text { (Mantel } \\
\text { Hanszel) }\end{array}$} & \multirow{2}{*}{ p-value } \\
\hline & Núm. & $\%$ & Núm. & $\%$ & & & & \\
\hline $\mathrm{NO}^{*}$ & 71 & 71,72 & 90 & 90,91 & 1,00 & & & \\
\hline SI & 28 & 28,28 & 6 & 6,06 & 5,92 & $(2,17-16,75)$ & 16,37 & 0,0000052 \\
\hline Número de compañeros & Núm. & $\%$ & Núm. & $\%$ & & & & \\
\hline Solo Uno & 44 & 44,44 & 60 & 60,61 & 1,00 & & & \\
\hline Más de Uno & 45 & 45,45 & 39 & 39,39 & 1,57 & $(0,85-2,93)$ & 2,35 & 0,125 \\
\hline Número de compañeros & Núm. & $\%$ & Núm. & $\%$ & & & & \\
\hline Solo uno & 44 & 44,44 & 60 & 60,61 & 1,00 & & & \\
\hline 2 a 3 & 37 & 37,37 & 36 & 36,36 & 1,40 & $(0,78-2,80)$ & 1,68 & 0,194 \\
\hline 4 a 5 & 12 & 12,12 & 1 & 1,01 & 16,36 & $(2,03-341,37)$ & 11,23 & 0,0008 \\
\hline 6 a 7 & 6 & 6,06 & 2 & 2,02 & 4,09 & $(0,83-34,29)$ & 4,04 & 0,044 \\
\hline
\end{tabular}

ción fue entrevistada, en relación a los antecedentes ginecológicos, obstétricos y socio culturales (Tabla 1).

Se realizó un análisis bivariado con los factores de riesgo Ginecoobstétricos y efectos en la población objeto de investigación, este análisis consiste en determinar, a partir del análisis bivariado, las relaciones existentes entre los factores de riesgo y la posibilidad de desarrollar lesiones preneoplásicas del cuello uterino, para establecer si existe asociación (significación estadística) entre el hecho de padecer la enfermedad y los factores de riesgo seleccionados mediante el estadígrafo chi-cuadrado de independencia (X2). Para estimar el riesgo relativo de padecer la enfermedad asociada con los factores de riesgo, se calculó la razón de productos cruzados (RPC) u odds ratio (OR).

\section{Análisis estadística}

Se analizaron algunos factores de riesgo relacionados con la historia reproductiva, en el cual reporto que la edad media de la menarquía fue de 13 años en los casos y 12,6 en los controles, en referencia al inicio de la actividad sexual la media es de 16,3 años; tanto para los casos como para los controles; en el grupo de los casos el número de compañeros sexuales varía entre los grupos presentándose una media de 2,2 compañe- ros sexual para los caos y de 1,5 para los controles.

Analizados las variables, relacionadas con los factores de riesgos ginecoobstétricos, existe significancia estadística en las variables de infección vaginal, ya que el riesgo de presentar lesiones preneoplásicas en el útero, se incrementan en 5,92 veces más en comparación de las que no han presentado infección, con un IC 95\% se encuentra $(2,17$ y 16,75), el valor de p 0,0000052), como también en la variable número de compañeros sexuales, las que han presentado entre 6 a 7 compañeros sexuales, incrementa el riesgo en 4,67 veces más en comparación a las que han tenido un solo compañero sexual; el rango de compañeros sexuales de 4 a 5, el cual tiene un OR de 16, el intervalo de confianza se encuentra entre $(2,03$ y 341,37$)$, lo que demuestra una amplia dispersión.

\section{Discusión}

Las mujeres con menor nivel académico acuden menos a la práctica de toma de la citología, lo que está ligado con el grado de conocimientos generales en salud. Esto tiene implicaciones críticas, pues en la medida que la mujer no sepa y entienda las razones para las cuales se requiere la toma periódica de citologías hay dificultades para que acuda regularmente a sus controles o cumpla 
con las instrucciones en caso de lesiones de bajo grado. De ahí que los estudios han evaluado las barreras para la toma de citología, además de las propiamente administrativas, se encuentre el desconocimiento de las pacientes y planteen que una de las estrategias clave es el desarrollo de programas de educación de usuarias para que identifiquen la importancia de realizarse el procedimiento, en nuestro estudio, en referencia a los diferentes grados de escolaridad, el 77,78\% de los casos y el $36,36 \%$ de los controles, han estudiado la primaria y el $4,04 \%$ de los caos y el $60,61 \%$ de los controles no tienen ningún grado de escolaridad formal, lo que corrobora lo anterior.

Existe una relación directamente proporcional entre el riesgo de lesión intraepitelial y el número de parejas sexuales. Esta exposición se ha relacionado básicamente con la probabilidad de exposición al VPH. En nuestro estudio el riesgo es 4,67 veces más en comparación a las que han tenido un solo compañero sexual, y estudios realizados por French PW, Coppleson M, refiere que "se ha observado que algunas proteínas básicas del semen humano pueden alterar las células epiteliares y subepiteliares del cérvix e inducir su transformación neoplásica y demostró que la protamina del semen humano y animal, produce en cultivos la transformación de las células epiteliares del cuello uterino humano"(8).

"Se ha demostrado la asociación de cáncer de cuello uterino con enfermedades de transmisión sexual tipo sífilis o blenorragia, así como historia de infección a repetición del aparato genital"(9); sin embargo, "no se ha demostrado relación con antecedentes de infección por Chlamydia trachomatiso herpes simplex"(10). En un estudio realizado en "tres países se encontró asociación entre infección por VPH y C. trachomatisen Colombia y España, pero no en Brasil"(11), mientras que en nuestro estudio, el presentar infección vaginal incrementa, en 5,88 veces más en comparación de las que no han presentado infección.

Según Félix J. Sansó Soberats, en su investigación titulada, Mortalidad por cáncer en Cuba, refiere que "Al modificar positivamente los factores de riesgo para el cáncer"(12) por ello "se estará incidiendo también sobre la expresión de otras enfermedades crónicas no trasmisibles, por lo que en muchas instancias, la prevención del cáncer es justamente uno de los resultados de los beneficios de buenas prácticas de salud"(13).

\section{Conclusiones}

Las mujeres que han presentado infecciones vaginales tienen un riego mayor en 5,92 veces en comparación de las que no han presentado dicha enfermedad, al igual que el tener más de cinco compañeros sexuales incrementa el riego de cáncer cervicouterino en 20,67 veces más en comparación con las que han presentado de 2 a 4 compañeros sexuales, nivel de escolaridad aumenta 2,24 el riesgo de padecer cáncer cervicouterino, lo que está ligado con el grado de conocimientos generales en salud.

\section{Referencias Bibliográficas}

1. Revista Colombiana de Obstetricia y Ginecología, ISSN 0034-7434 versión impresa.

2. Ibíd.

3. Wang SS, Wheeler CM, Hildesheim A, Schiffman M, Herrero R, Bratti MC, et al. 2001. Human leukocyte antigen class I and II alleles and risk of cervical neoplasia: results from a population-based study in Costa Rica. J Infect Dis 184:1310-1314.

4. Sánchez V, Izquierdo A, Beltrán M, Viladiu P. 1997. Epidemiología del cáncer invasor de cerviz en el área sanitaria de Girona durante el período 1980-1989. Registro poblacional de cáncer de Gerona. Rev Esp Salud Pública 71:19-26.

5. Hart KW, Williams OM, Thelwell N, Fiander AN, Brown T, Borysiewicz LK, et al. 2001. Novel method for detection, typing and quantification of human papillomaviruses in clinical samples. J Clin Microbiol 39:32043212.

6. Ellerbrock TV, Chiasson MA, Bush TJ, Sun XW, Sawo D, Brudney K, et al. 2000. Incidence of cervical squamous intraepithelial lesions in HIV-infected women. JAMA 283:1031-1037.

7. Szklo M, Nieto J. 2003. Epidemiología Intermedia. Ediciones Diaz Santos, p. 26.

8. French PW, Coppleson M, Reid BL. 1987. Effects of sperm protamine on human cervical epithelial cells and BHK 21 cells in vitro. JR Soc Med 80:434-437. 
9. Varghese C, Amma NS, Chitrathara K, Dhakad N, Rani P, Malathy L, et al. 1999. Risk factors for cervical dysplasia in Kerala, India. Bull World Health Organ 77: 281-3.

10. Molano M, Posso HJ, Weiderpass E, van den Brule AJ, Ronderos M, Franceshi S, et al. 2002. Prevalence and determinants of HPV infection among Colombian women with normal citology. Br J Cancer 87:324-333.
11. Munoz N, Kato I, Bosch FX, Eluf-Neto J, de San Jose S, Ascunce N, et al. 1996. Risk factor for HPV detection in middle-age women. Sex Transm Dis 23:504-510.

12. Félix J. Sansó Soberats; Patricia Alonso Galbán; Rosa María Torres Vida. 2010. Mortalidad por cáncer en Cuba.

13. Bernard W, Stewart and Alan S. Coates. 2005. Cancer prevention: a global perspective. J Clin Oncol 23:392-403. 


\title{
POSGRADOS CLÍNICOS
}

\section{Universidad}

Surcolombiana

\section{ESPECIALIZACIÓN EN EPIDEMIOLOGÍA}

Registro ICFES No. 111456140404100113100

Creada mediante acuerdo Consejo Superior Universitario No. 009 del 10-05-1999

Registro calificado de calidad Res. No 3299 del 3-06-2008, Ministerio de Educación Nacional.

$\begin{array}{ll}\text { Denominación Académica: } & \text { Programa de Especialización en Epidemiología } \\ \text { Modalidad: } & \text { Semipresencial } \\ \text { Duración: } & 1 \text { año } \\ \text { Cupos: } & 30 \text { Anuales } \\ \text { Título: } & \text { Especialista en Epidemiología }\end{array}$

\section{ESPECIALIZACIÓN EN ENFERMERÍA NEFROLÓGICA Y UROLÓGICA}

Registro calificado MEN No. 9906 de Diciembre 23 de 2008.

\author{
Denominación Académica: Programa de Especialización en Enfermería Nefrológica y urológica \\ Modalidad: \\ Presencial \\ Duración: \\ 3 semestres \\ Cupos: \\ 6 Anuales \\ Título: \\ Especialista en Enfermería Nefrológica y urológica
}

\section{ESPECIALIZACIÓN EN ENFERMERÍA CUIDADO CRÍTICO}

Registro calificado MEN No. 9905 de Diciembre 23 de 2008.

$\begin{array}{ll}\text { Denominación Académica: } & \text { Programa de Especialización en Enfermería Cuidado Crítico } \\ \text { Modalidad: } & \text { Presencial } \\ \text { Duración: } & 3 \text { semestres } \\ \text { Cupos: } & 6 \text { Anuales } \\ \text { Título: } & \text { Especialista en Enfermería Cuidado Crítico }\end{array}$

